\title{
Understanding the Determinants of Cloud Computing Adoption for High Performance Computing
}

\author{
Theo Lynn \\ Dublin City University \\ theo.lynn@dcu.ie \\ John P. Morrison \\ University College Cork \\ j.morrison@,cs.ucc.ie
}

\author{
Xiaoning Liang \\ Dublin City University \\ xiaoning.liang@tcd.ie \\ Grace Fox \\ Dublin City University \\ grace.kenny@,dcu.ie
}

\author{
Anna Gourinovitch \\ Dublin City University \\ anna.gourinovitch@dcu.ie \\ Pierangelo Rosati \\ Dublin City University \\ pierangelo.rosati@dcu.ie
}

\begin{abstract}
Within the complex context of high performance computing (HPC), the factors influencing technology adoption decisions remain largely unexplored. This study extends Diffusion of Innovation (DOI) and Human-Organization-Technology fit (HOT-fit) theories into an integrated model, to explore the impact of ten factors on cloud computing adoption decisions in the HPC context. The results suggest that adopters and non-adopters have different perceptions of the indirect benefits, adequacy of resources, top management support, and compatibility of adopting cloud computing for HPC. In addition, perceptions of the indirect benefits and HPC competences can be used to predict the cloud computing adoption decision for HPC. This is one of the first studies in the information systems (IS) literature exploring the factors impacting the cloud computing adoption decision in the important context of HPC. It integrates two influential technology adoption theories and enhances understanding of the key factors influencing organizations' cloud computing adoption decisions in this context.
\end{abstract}

\section{Introduction}

Cloud computing is, without doubt, one of the major emergent paradigms in information systems research and practice. The National Institute of Standards and Technology (NIST) defines cloud computing as a model for "enabling convenient, ondemand network access to a shared pool of configurable computing resources (e.g. networks, servers, storage, application, and services) that can be rapidly provisioned and released with minimal management effort or service provider interaction" [1-
2]. Attracted by the promise of information technology (IT) efficiencies and increased business agility, enterprises are incorporating cloud computing in their IT strategies [3-4]. Despite the extensive literature on the benefits of cloud computing, our understanding of cloud computing adoption decisions is marred by inconsistencies in the influence of a myriad of organizational, technological, environmental, and human factors [5-8], which vary by situational context [4, 9-10]. Thus, in highly complex and under researched contexts such as high performance computing (HPC), there is a need for research which elucidates the role of the various organizational, technological, environmental and human factors.

Advances in the design of higher performance processors, functional accelerators, interconnects, and associated software have resulted in increasingly powerful computers measured in petascale terms, often referred to as supercomputing or HPC [12]. HPC can be defined as the coordinated use of massive parallel architectures for the solution of a single problem and are characterized by the scale of compute power required to process a job quickly [13]. The emphasis in HPC is on performance and vertical scaling. HPC-oriented problems are typically computationally and data intensive simulation and analytics problems [11]. Such advanced computing is costly in terms of upfront capital investment and direct and indirect operational expenditure as HPC requires significant energy and expertise to operate [12]. Due to the cost of HPC, it is unsurprising that the focus of such computing power has traditionally been on high value scientific or technical computing workloads characterized by specialist software and the need for high reliability and resiliency. HPC is commonly used in automotive and aerospace product development, oil and gas exploration, genomics and drug discovery, weather prediction and climate modeling, 3D image rendering, and complex financial modeling [11]. Thus, 
access to HPC infrastructure has typically been limited to large mature organizations, government research centers or agencies, and universities [11]. A recent IDC report for the European Commission highlights the importance of HPC:

"The use of high performance computing (HPC) has contributed significantly and increasingly to scientific progress, industrial competitiveness, national and regional security, and the quality of human life. HPC-enabled simulation is widely recognized as the third branch of the scientific method, complementing traditional theory and experimentation. HPC is important for national and regional economies-and for global ICT collaborations in which Europe participatesbecause HPC, also called supercomputing, has been firmly linked to accelerating innovation" [11, pp.20].

Geist and Reed [12] note that large-scale commercial cloud computing infrastructures share common scales with large scale HPC systems with both services driven by cost, reliability, and energy efficiency imperatives. However, they also note important differences. Cloud Computing requires justin-time system deployment with minimal time for onsite configuration and testing, energy minimization and cost-effective cooling, and due to competitive forces, higher tolerances for hardware component failures; factors that are not consistent with the expectations of the HPC community for whom performance is key [12]. The emphasis in cloud computing is on scalability, in this case horizontal scaling through the addition of more machines, in contrast to vertical scaling in HPC. Against this background, the rate of cloud computing adoption for HPC has been lower than other areas [14]. Compared to the wider information and communication technology (ICT) market, cloud computing makes up a relatively small proportion of overall revenues for the HPC market but as a segment has begun to show evidence of strong growth [11].

Despite the societal impact of high performance computing, research on HPC in the IS literature, and specifically HPC in the cloud, is limited [15-16]. To the best of our knowledge, this is the first study to examine the adoption of cloud computing for high performance computing (HPC), a small but high value segment of the ICT market [11]. It differs from other cloud computing contexts in that the emphasis in HPC is vertical scalability rather than horizontal scalability and tightly coupled rather than loosely coupled (or socalled 'embarrassingly parallel') workloads. Given the emphasis on performance over scalability in HPC, it is worth exploring whether the determinants for cloud adoption differ for HPC workloads.

HPC infrastructure has traditionally been unavailable to many organizations due to its high cost and the specialized technical knowledge required to configure and maintain such infrastructure. In line with Beck and Toenker [15], we posit that HPC in the cloud allows organizations, particularly small-tomedium enterprises, to access HPC infrastructure on a more cost-effective basis and as a result better compete by overcoming internal constraints to generate new capabilities, enter new markets, adopt new resources, and develop new products. This may enable an acceleration of innovation resulting in a positive impact for industry and society as a whole. With this in mind, the primary objective of this study is to elucidate the factors which drive or inhibit cloud computing adoption for HPC. In focusing on HPC, the paper makes an important contribution to the literature on cloud computing, HPC and the adoption process.

The paper is organized as follows. In the next section, we discuss the related literature in the cloud computing context. Two pertinent theories are integrated to develop the research model and hypotheses in Section 3. This is followed by an overview of the methodology employed. Section 5 presents the analysis and is followed by a discussion of the findings. Limitations and opportunities for future research are discussed in the final section.

\section{Literature Review}

\subsection{Cloud Computing Adoption}

The information systems literature on innovation adoption is well-established and comprises a number of highly cited frameworks. A recent review of the literature on cloud computing adoption found that many studies fail to utilize these guiding frameworks [1]. Furthermore, the studies which adopt a guiding framework lack a unified approach to measuring adoption with studies leveraging Diffusion of Innovation (DOI), Technology-OrganizationEnvironment (TOE), Human-OrganizationTechnology fit (HOT-fit) theory, Information Processing View (IPV), and Technology Acceptance Model (TAM) to understand adoption in the cloud computing context.

For the purpose of this study, each framework was reviewed to explore their relevance when examining cloud computing adoption decisions in HPC computing. First, DOI theory proposed by Rogers [16] describes an innovation as an idea, practice, or object that is perceived as new to an individual or 
organization [17]. DOI theory holds that the technology adoption decision can be explained by five innovation characteristics: (i) relative advantage, (ii) compatibility, (iii) complexity, (iv) observability, and (v) trialability. Rogers [17] embeds innovation adoption within the context of a social system and suggests that the individual, the internal organizational structure, and external characteristics of the organization influence adoption. Studies have shown support for the application of DOI in the cloud computing adoption context, with perceived indirect benefits, the complexity and computability of cloud computing technologies, and an organization's business needs all found to influence cloud computing adoption by an organization [4, 8, 19].

Similar to DOI, the TOE Framework proposed by Tornatzky and Fleischer [20] suggests that IT adoption is influenced by three contexts - (a) technological, (b) organizational, and (c) environmental. These contexts are consistent with DOI theory in that the technological context incorporates the innovation characteristics proposed by Rogers [17]. TOE theory also explores the influence of organizational factors, such as firm size, technology readiness, employee competence, and top management support $[6,9,10$, 18]. Put differently, TOE theory extends DOI and incorporates environmental factors to explain technology adoption intention $[4,6,21]$. In support of TOE, studies find that perceived benefits, top management support, competition, partner pressure, business concerns, and the firm's IT capability can influence cloud computing adoption $[5,6,9,10]$.

In congruence with DOI and TOE, the HOT-fit model, proposed by Yusof et al. [22], asserts that organizational and technological factors are determinants of successful IS adoption. The HOT-fit model incorporates organizational and technological dimensions with human factors, claiming that user attitude and competences also have a positive impact on technology adoption [22]. Drawing on HOT-fit, Lian et al. [10] find that in addition to organizational and technological factors (e.g., data security and technological complexity), human factors (i.e., perceived technical competence) can also influence cloud computing adoption by an organization.

IPV argues that organizations possess both a need and capacity to process information in order to support decision-making and reduce uncertainty [23-24]. Applying IPV to the cloud computing context, Cegielski et al. [25] find that an organization's information processing requirements and capabilities

\footnotetext{
1 An exploratory Delphi study was conducted with HPC decision makers to explore the relevance of organizational, environmental and individual factors in this context.
}

interact to influence cloud computing adoption in supply chains. The TAM model [26] suggests that perceived ease of use and perceived usefulness of new technologies are primary facilitators of acceptance and usage among employees [21, 27]. In support of TAM, Gangwar et al. [21] demonstrate that perceived usefulness and perceived ease of use are positively related to cloud computing adoption.

The existing literature suggests that the influential factors in cloud computing adoption decisions can be categorized into four groups: (i) human factors such as the personal innovativeness of key decision makers, perceived technical competence and training [10, 22]; (ii) organizational factors including adequacy of resources, top management support, perceived indirect benefits, and relative advantage $[4,10,19,21]$; (iii) technological factors such as perceptions of the innovation's complexity, compatibility, and security $[5,7,28]$; and (iv) environmental factors such as competitive pressure, government policy, and partner support $[4,10,21]$.

\subsection{DOI and HOT-Fit frameworks}

While there is no dominant model for exploring technology adoption in the cloud computing context, there are many similarities among the theories and frameworks operationalized within this context. Furthermore, the trend of combining numerous frameworks or theories is evident with the majority of researchers integrating two or more theories to gain a more comprehensive understanding of adoption in the cloud computing context. This approach is appropriate due to complementarity of many of the popular technology adoption theories. For instance, DOI, TOE, and HOT-fit theories complement each other, to provide a comprehensive understanding of the key determinants of technology adoption $[4,7,10]$.

The aim of this research is to develop an understanding of technology adoption decisions in the complex context of high performance computing, with a focus on decisions to adopt cloud computing. To achieve this aim, we leverage DOI theory and the HOT-fit framework, as these two theories are commonly used to explain technology adoption and provide a more holistic understanding of cloud computing adoption [8-10] ${ }^{1}$. Another reason for applying DOI theory rather than TOE theory is that prior research offers mixed support for the importance of environmental factors in the cloud computing context $[1]^{2}$. We also conducted an exploratory survey

\footnotetext{
2 Environmental factors were not viewed as important factors in our exploratory study.
} 
on key drivers and barriers of cloud computing adoption in the HPC context. The results show support for human, organizational, and technological factors but not for the environmental factors. For instance, government policy was neither a barrier (2.83 out of 5 ) nor a driver (2.31 out of 5) of cloud computing with very low variance in response. These results are line with some prior studies applying TOE theory which found environmental factors did not influence cloud computing adoption $[4,6]$. Thus, DOI theory is more applicable in this context. As noted above, DOI theory argues that top management characteristics and internal organizational structure can influence end users' adoption intentions towards cloud computing. Adding the HOT-fit framework, we posit that human, technological, and organizational factors can influence cloud computing adoption by an organization.

While the extant literature supports the influence of many innovation and organizational characteristics on technology adoption decisions, the DOI theory asserts that the salient innovation characteristics vary across contexts [29]. For instance, a recent Taiwanese study found that relative advantage was significantly related to cloud computing adoption, whereas complexity and compatibility did not affect adoption [6]. In contrast, a study conducted among a Portuguese sample, found that both relative advantage and complexity significantly influenced cloud computing adoption, while compatibility had no impact [4]. Thus, it is recommended that researchers consider the research context when deciding on the appropriate technology adoption model and factors [8].

\section{Research Model and Hypotheses}

This study draws on DOI and HOT-Fit to develop the framework and hypotheses proposed in Figure 1.

\subsection{Human factors}

Personal innovativeness denotes the openness of an individual to new technology. Studies show that an individual's perceived level of innovativeness can influence how they respond to new technologies [10, 30]. Thus, personal innovativeness may predict whether a person intends to adopt an innovation earlier than others $[5,30]$. This study asserts that if an individual (key decision maker) is open to innovation, he/she will be more likely to adopt cloud computing for HPC. Hence, we present the following hypothesis:

H1: Personal innovativeness is positively related to the adoption of cloud computing for HPC.

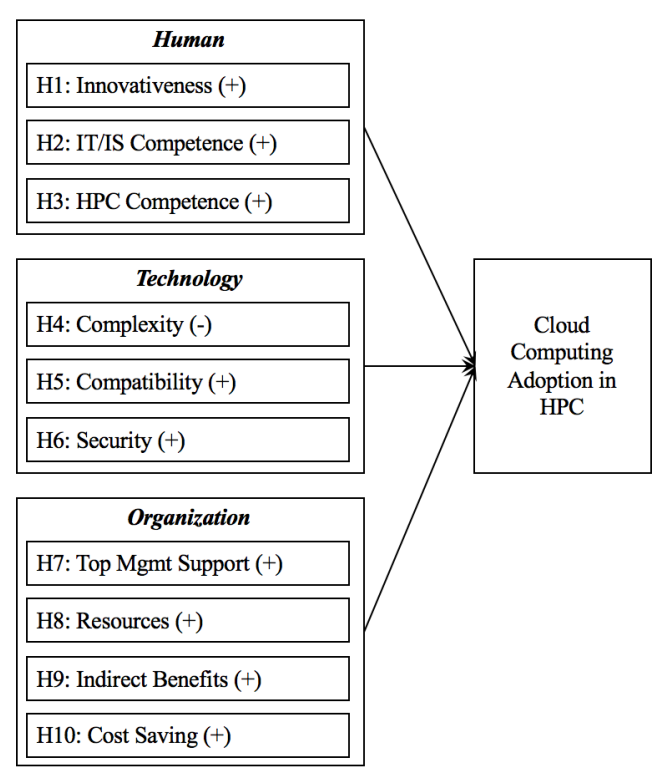

Figure 1. Proposed framework

Two human factors related to an organization's technological readiness may also positively influence adoption $[4,5]$. In order to enhance technological readiness, organizations are required to have specialized human resources (e.g. competent HPC staff and/or IT/IS staff) who have the knowledge and skills to implement cloud computing for HPC. Thus, employees' technological competencies are believed to have a positive impact on cloud computing adoption for HPC workloads. Studies show that IT expertise is essential for organizations that intend to adopt cloud computing $[5,10,21]$. Since cloud computing is still a new concept for organizations, it requires technical skills to ensure its successful implementation [19]. Whilst this expertise may vary depending on the type of cloud computing service to be consumed (SaaS, $\mathrm{PaaS}$ or IaaS), we posit that technical competence would be a significant determinant of adoption for HPC, as organizations using HPC often use proprietary, tightly configured, or specialist software requiring specialist expertise. Organizations with higher levels of HPC and IT competencies are likely to be more confident in their technical decisionmaking and more likely to implement cloud computing for HPC. Thus, we propose:

H2: IT/IS competence is positively related to the adoption of cloud computing for HPC.

H3: HPC competence is positively related to the adoption of cloud computing for HPC.

\subsection{Technological factors}

Complexity represents organizations' perceived difficulty in the understanding and usage of an 
innovation or new technology [18]. Organizations tend to adopt new technologies that are easy to use, as complex technologies reduce perceptions of the usefulness and ease of use, resulting in decreased adoption [21]. In addition, the complexity of the technology may increase the perceived risk associated with its implementation. If organizations consider cloud computing for HPC as complex and challenging, they are less likely to adopt, due to the lack of confidence in their ability to understand and implement the system [6]. Therefore, we posit:

H4: Complexity is negatively related to the adoption of cloud computing for HPC.

Compatibility refers to "the degree to which an innovation is perceived as consistent with the existing values, past experiences, and needs of potential adopters" [6, p.240]. Organizations are less likely to adopt cloud computing if it is not compatible with these values, as adoption may require major adjustments in organizational processes, considerable learning, and tremendous commitment from stakeholders [6, 31]. Many studies describe compatibility as a key driver of technology adoption $[17,31]$. For instance, Gangwar et al. [21] and Alshamaila et al. [5] both argue that the compatibility between cloud computing and the existing technologies of an organization, in this case HPC, increases the perceived usefulness and ease of use, thus increasing adoption intentions. Thus, we propose:

H5: Compatibility is positively related to the adoption of cloud computing for HPC.

Security concerns are consistently reported as a major barrier to cloud computing adoption [11, 33]. Typically, these security concerns relate to insecure interfaces or data loss [6]. Security is perceived to directly relate to the reliability of a cloud computing system and is positively associated with the cloud computing adoption decision with higher perceptions of security increasing adoption intentions [28]. Organizations are more likely to adopt cloud computing for HPC if they deem cloud computing as a reliable and secure platform. Thus, we hypothesize:

H6: Perceived security is positively related to the adoption of cloud computing for HPC.

\subsection{Organizational factors}

Top management support is conceptualized as the commitment of top management to the adoption of cloud computing [10]. The adoption of cloud computing requires the integration of resources and a supportive environment [34]. Top management involvement in the cloud computing adoption decision and implementation process is essential as it guarantees that sufficient resources will be allocated to support implementation [5]. In addition, when top management shows support for cloud computing, it is more likely that the value of adopting will be communicated throughout the organization [6]. Consequently, a shared vision will be created within the organization, enabling the adoption of cloud computing for HPC. Thus, we propose:

H7: Top management support is positively related to the adoption of cloud computing for HPC.

Adequate resources refer to the resources needed for the adoption of cloud computing. Previous studies show that organizations with the necessary resources are more likely to adopt cloud computing than those with inadequate levels of resourcing [35]. Cloud computing adoption for HPC is a large project that requires top management commitment, ample time, sufficient money, competent human resources, and technological competencies $[10,36]$. In this case, adequate resources give organizations the confidence to invest in cloud computing for HPC.

H8: Adequate resources are positively related to the adoption of cloud computing for HPC.

Extant literature suggests that adopting cloud computing provides organizations with direct and indirect benefits. We suggest that this is also true for HPC use cases. For instance, the adoption of cloud computing enables organizations to gain access to hardware, software, or other ICT infrastructure not available in their own datacenters [9, 37]. Such advantages are termed direct benefits. In addition to these direct benefits, organizations are also motivated by the indirect benefits associated with the adoption of cloud computing. These include strategic benefits, such as improving the organizational image, competitive advantage, or relationships with customers or business partners [37-38]. We posit that, the more indirect benefits organizations can obtain from cloud computing adoption for HPC, the more likely they will be to adopt cloud computing for HPC.

H9: Indirect benefits are positively related to the adoption of cloud computing for HPC.

As cloud computing allows organizations to use certain services through the Internet, organizations do not necessarily have to spend tremendous money on building, maintaining, or upgrading their information systems. Thus, adoption allows organizations to reduce upfront capital expenditure and operational costs, thereby enabling them to gain a cost reduction advantage $[35,39]$. Studies show that cost reduction is positively associated with organizations' perceptions of the ease of use and convenience of adopting cloud 
computing, thus increasing adoption intention [9, 28]. HPC typically requires significant upfront capital expenditure. In addition, as HPC tends to push performance at all costs, HPC infrastructure has extremely high operating costs compared to more general IT. For example, the top 500 supercomputers consume between 1.3 megawatts and 15.3 megawatts of power [40]. This is not only a high cost from an energy perspective but also has a substantial environmental impact. Thus, we posit that cost efficiencies will influence adoption.

H10: Cost reduction is positively related to the adoption of cloud computing for HPC.

\section{Methodology}

A questionnaire was designed to test the proposed framework (Copy available upon request). All items were sourced from the literature and adapted to fit the context of HPC. Innovativeness (four items), HPC competence (six items), IT/IS competence (five items), complexity (five items), and adequate resources (four items) were adapted from Lian et al. [10]. The six items used to measure indirect benefits were adapted from Kuan and Chau [38] and Lian et al. [10]. Top management support (three items) and compatibility (four items) were adapted from Premkumar and Roberts [31] and [10]. The four items to measure reliability and security were adapted from Soliman and Janz [41] and the three cost reduction variables were adapted from Lian et al. [10] and Soliman and Janz [41]. Each item was measured on a five-point Likert scale. Given the small size of the HPC market, a database was developed to recruit HPC decision makers, which included C-suite employees across various industries from oil and gas to genomics, as well principal investigators and research group heads at Universities. Decision makers were identified through an online search. The survey was distributed to 619 HPC decision-makers worldwide using publically available email addresses.

Ninety-eight participants started the survey, with 92 complete responses. This represented a response rate of $15.83 \%$, which was deemed adequate given the relatively small number of organizations in the HPC market. Among the sample, 57.60\% are based in the European Union, $35.90 \%$ in North America and the remainder worldwide. Respondents are from different organizational contexts including academic (66.30\%), commercial (30.40\%), and government (5.40\%). Among these respondents, $47.8 \%$ indicate that they have adopted cloud computing for HPC in their organization, while $52.2 \%$ indicate they have not yet adopted cloud computing.
Prior to the data analysis, non-response bias and exploratory factor analysis (EFA) were conducted. Since the response rate was slightly lower than the recommended threshold (36+/-13) [42], non-response bias was tested in order to check the representativeness of the responses in this study. Following Wilcox et al. [43], we tested the non-response bias by comparing the organizational variables from the early respondents with the late respondents. The number of HPC users, the weekly HPC usage, and the respondent's familiarity with HPC were used as benchmarking organizational variables. The t-test results showed that there was no significant difference between early and late respondents across any of these variables. Therefore, the sample was deemed representative. Exploratory factor analysis was carried out in SPSS 23 to test the reliability and validity of the measures for all key constructs in the new context of HPC. Factor analysis with principal-component factoring method and VARIMAX rotation was used to test the discriminant and convergent validity. The Kaiser-Meyer-Olkin (KMO) values of the three dimensions were all above the threshold of .70, indicating that these items were suitable for conducting factor analysis. Three factors were generated under the human dimension; personal innovativeness, HPC technical competence, and IT/IS technical competence. Three factors were also generated under the technology dimension; complexity, compatibility, and security. One item from the security construct was dropped to increase construct reliability. Four factors were generated under the organization dimension; indirect benefits, adequate resources, top management support, and cost reduction. All the Cronbach's alpha values were larger than .60, suggesting that the measures were reliable [44]. All items loaded onto their expected construct with factor loadings above .60. Thus, convergent validity and discriminant validity for each construct was also achieved. The composite scores of all factors were calculated for further data analysis. As shown in Table 1, respondents rank innovativeness, compatibility, complexity, indirect benefits, and cost reduction as the most critical factors influencing cloud computing adoption.

Table 1. Descriptive statistics

\begin{tabular}{ccccc}
\hline Dimension & Factor & Mean & Std.D & Rank \\
\hline Human & Innovativeness & 3.80 & .65 & 1 \\
& HPC competence & 3.28 & .97 & 7 \\
& IT/IS competence & 2.87 & .98 & 10 \\
\hline Organization & Benefits & 3.39 & .72 & 4 \\
& Adequate resources & 2.88 & .87 & 9 \\
& Cost reduction & 3.35 & .90 & 5 \\
& Top management & 2.90 & .87 & 8 \\
& support & & & \\
\hline Technology & Compatibility & 3.54 & .78 & 2 \\
& Security & 3.29 & .83 & 6 \\
& Complexity & 3.46 & .83 & 3 \\
\hline
\end{tabular}


To compare the perceptions of adopters and non-adopters, a series of T-tests were conducted. The results presented in Table 2 suggest that adopters and non-adopters have different perceptions of four factors, namely indirect benefits, adequacy of resources, top management support, and compatibility. Unsurprisingly, these mean differences show that adopters perceive the adoption of cloud computing for HPC more positively than nonadopters.

Table 2. T-test comparison of adopters and non-

\begin{tabular}{llll}
\multicolumn{4}{c}{ adopters } \\
\hline & Adopters & $\begin{array}{l}\text { Non- } \\
\text { adopters }\end{array}$ & T-value \\
\hline Innovativeness & $3.83(.68)$ & $3.78(.62)$ & -.36 \\
HPC competence & $3.44(.99)$ & $3.13(.94)$ & -1.53 \\
IT/IS competence & $2.93(.98)$ & $2.80(.98)$ & -.62 \\
Complexity & $3.43(.93)$ & $3.49(.73)$ & .37 \\
Compatibility & $3.78(.70)$ & $3.31(.80)$ & $-2.97 * *$ \\
Security & 3.36 & $3.23(.66)$ & -.76 \\
& $(1.00)$ & & \\
Top management & $3.15(.81)$ & $2.67(.85)$ & $-2.78^{* *}$ \\
support & $3.09(.94)$ & $2.69(.76)$ & $-2.26^{*}$ \\
$\begin{array}{l}\text { Adequate } \\
\text { resources }\end{array}$ & & & \\
Indirect benefits & $3.71(.65)$ & $3.10(.66)$ & $-4.46^{* *}$ \\
Cost reduction & $3.52(.87)$ & $3.19(.90)$ & -1.75 \\
\hline Numbers in brackets are standard deviations; ${ }^{* *} \mathrm{p}<.01 ;{ }^{*} \mathrm{p}<.05$.
\end{tabular}

\section{Hypothesis Testing}

To empirically test the framework, logistical regression analysis was conducted in SPSS 23 with all ten factors. The variance inflation factors (VIF) and tolerance values were calculated to test the multicollinearity of these factors. VIF scores ranged from 1.09 to 2.19 , all below the threshold of 3 . The tolerance values ranged from .46 to .92 , all above the cut-off score of .10. These results suggest that multicollinearity is not an issue in this study [44].

Table 3. Logistic regression results

\begin{tabular}{|c|c|c|c|c|c|c|}
\hline & $\mathrm{B}$ & S.E. & Wald & Sig. & $\begin{array}{l}\text { Collinearit } \\
\text { Tolerance }\end{array}$ & $\begin{array}{l}\mathrm{y} \\
\mathrm{VIF}\end{array}$ \\
\hline Innovativeness & -.31 & .41 & .55 & .46 & .92 & 1.09 \\
\hline HPC competence & $.70^{*}$ & .36 & 3.76 & .05 & .58 & 1.72 \\
\hline IT/IS competence & -.70 & .37 & 3.51 & .06 & .55 & 1.84 \\
\hline Complexity & -.17 & .34 & .26 & .61 & .83 & 1.21 \\
\hline Compatibility & -.12 & .45 & .07 & .80 & .50 & 1.99 \\
\hline Security & -.45 & .39 & 1.37 & .24 & .67 & 1.49 \\
\hline $\begin{array}{l}\text { Top management } \\
\text { support }\end{array}$ & .37 & .40 & .86 & .36 & .46 & 2.19 \\
\hline Adequate resources & .37 & .40 & .86 & .35 & .48 & 2.08 \\
\hline Indirect benefits & $1.87 * *$ & .59 & 9.88 & .00 & .86 & 2.09 \\
\hline Cost reduction & -.21 & .35 & .36 & .55 & .62 & 1.61 \\
\hline Constant & $-4.50 \dagger$ & 2.56 & 3.09 & .08 & & \\
\hline
\end{tabular}

Model Evaluation. The omnibus test of model coefficients explores whether the independent variables in the model can explain the variation in the dependent variable. A significant result suggests that the independent variables can improve the prediction of the dependent variables. In this case, a chi-square $\chi^{2}$ of 26.27, with a degree of freedom of 10 and a significance value below $.01(p=.00)$ reveals that the ten factors of interest can significantly improve the prediction of the cloud computing adoption decision.

Goodness-of-fit statistics. The Hosmer and Lemeshow test gives a $\chi^{2}$ (8) of 2.98, with a significance value of .94 . This non-significant result suggests an acceptable match between the predicted adoption decision and actual adoption decision. -2 log likelihood (-2LL) and Nagelkerke pseudo $\mathrm{R}^{2}$ were also calculated to show the power of the research model in explaining the data variation. The lower the $-2 \mathrm{LL}$ value is, the better the model fit is. In this case, a -2 log likelihood value of 98.45 was acceptable. Nagelkerke Pseudo $\mathrm{R}^{2}$ represents the amount of variation explained by the model. The model explains $34 \%$ of the variance in cloud computing adoption.

Statistical test for individual predictors. The Wald chi-square statistics were used to check the predictive ability of individual predictors. The results show that indirect benefits $(\mathrm{p}<.01)$ and HPC competence $(\mathrm{p}<$ $.05)$ are statistically significant predictors of cloud computing adoption. Thus, $\mathrm{H} 3$ and $\mathrm{H} 9$ were supported. Indirect benefits and HPC competence are positively related to an organization's likelihood of adopting cloud computing for HPC.

Discriminating power. The logistic regression analysis also reveals the predictive accuracy of the research model (Table 4). The model yields a correct prediction rate of $76.1 \%$ for non-adopters and of $72.7 \%$ for adopters. The overall correct prediction rate is $74.4 \%$. These results illustrate that these predictors have sufficiently higher discriminating power than the random choice model.

Table 4. Correct prediction results

\begin{tabular}{lllcr}
\hline & \multicolumn{2}{c}{ Not adopt } & Adopt & \% correct \\
\hline Baseline & Not adopt & 46 & 0 & 100 \\
& Adopt & 44 & 0 & 0 \\
& Overall \% & & & 51.1 \\
Final & Not adopt & 35 & 11 & 76.1 \\
& Adopt & 12 & 32 & 72.7 \\
& Overall \% & & & 74.4 \\
\hline
\end{tabular}

\section{Discussion}

Integrating DOI and the HOT-fit model, this study finds that only organizational and human factors significantly influence organizations' cloud computing adoption decisions for HPC. Specifically, 
decision makers' perceptions of indirect benefits and existing HPC competences predict their cloud computing adoption decisions with an overall correct prediction rate of $74.4 \%$.

Human factors. The results indicate that, even though innovativeness is high, this does not necessarily lead to cloud computing adoption. This finding is inconsistent with previous studies $[5,10]$. Descriptive statistics reported in Table 3 show that personal innovativeness is the top-rating factor of cloud computing adoption for HPC. Thus, the reasons behind this inconsistency merit further investigation. The results also suggest that organizations with superior HPC competences perceive themselves to possess more advanced computing abilities, and thus are more willing to adopt cloud computing [9]. The insignificant relationship between IT competences and adoption may be because organizations with sufficient IT expertise may have gone through some of the technological changes required for cloud computing, reducing the impact of technological competences on the cloud computing adoption but an increased effect on the extent of cloud computing implementation [6]. These mixed results are congruent with the inconclusive results reported in previous studies: some report a positive impact $[5,9,19,21]$, while others report insignificant results $[6,8]$. Human factors thus require further investigation.

Technological factors. None of the technological factors in this study; compatibility, complexity, and security influence the adoption of cloud computing for HPC by an organization. As suggested by previous studies, there may be alternative explanations for these results $[5,8,21]$. First, organizations may realize that the benefits of adopting cloud computing for HPC are likely to outweigh the perceived complexity or security concerns [7]. Thus, they may adopt cloud computing for HPC to maximize these perceived benefits. Second, if the organizations perceive themselves as having superior existing technological competences, they may adopt cloud computing regardless of the compatibility between cloud computing and their existing HPC systems or processes. Third, though cloud security remains a major concern [5], the technological advances in securing data privacy and confidentiality on cloud computing platforms may have given organizations confidence in implementing cloud services [4]. Thus, the previously anticipated technological factors may become less critical in determining cloud computing adoption for HPC.

Organizational factors. Only perceived indirect benefits significantly and positively influenced the cloud computing adoption decision for HPC. This is congruent with previous studies [7, 9, 21], suggesting that indirect benefits have a strong influence on adoption intention and decisions [28]. A useful implication arising from this is that cloud computing service providers should highlight the wider set of benefits associated with adopting cloud computing for HPC to their customers in order to increase their likelihood to adopt. Surprisingly, adequate resources and top management support are not found to influence cloud computing adoption for HPC. These results contradict many prior studies [4-6, 21]. Finally, cost reduction does not influence cloud computing adoption for HPC. This may be HPC-specific as HPC typically involves specialist computing and scientific expertise where the decision-making is devolved to those with the required expertise. Similarly, HPC has a high cost profile compared to general IT expenditure. This may also reflect a sampling characteristic. The sample comprises organizations operating in the traditional HPC market not SMEs. The use of cloud computing for cost efficiencies is a widely reported benefit for smaller or new enterprises [19]. While greater support for HPC in the cloud would expand the market for SMEs, larger organizations using HPC, have sufficient resources and are less likely to adopt cloud computing for the purpose of cost reduction.

\section{Conclusions}

Based on the views expressed by 92 HPC decisionmakers worldwide, this study finds that indirect benefits and HPC competences are key drivers of cloud computing adoption for HPC. The study makes three important contributions. First, the study incorporates DOI with HOT-fit theories to provide a holistic view of determinants of cloud computing adoption for HPC. By validating the positive role of human and organizational factors in influencing cloud computing adoption, this study provides some support for the extension of the DOI-HOT framework to explain cloud computing adoption decisions. Second, to the best of our knowledge, this study is the first to investigate the influential factors of cloud computing adoption in an HPC context. As indicated earlier, there is a dearth of research on cloud computing adoption in the HPC area. Thus, this study provides important insights and answers calls for clarification on the drivers and inhibitors of cloud computing adoption in differing contexts [1]. Third, this study provides insights for cloud service providers with findings suggesting that cloud service providers should emphasize the indirect benefits of adopting cloud computing for HPC in their communications to potential customers. The major limitations of this study are the use of single-informant and the small sample size. The results would be more robust with 
data from multiple respondents and from a larger sample. An additional round of data collection is currently underway with the aim of exploring the drivers of adoption among a larger, more diverse sample. Future research may also adopt a multipleinformant approach or test these factors among smaller companies who could leverage the cloud to expand into the area of HPC. Future studies could consider the possibility of including other determinants to provide a more sophisticated explanation or develop a domainspecific model. Detailed interview data may provide insights which explain the significant and insignificant results.

\section{Acknowledgement}

This work is partially funded by the European Union's Horizon 2020 Research and Innovation Programme through the CloudLightning project (http://www.cloudlightning.eu) under Grant Agreement Number 643946.

\section{References}

[1] Asatiani, A., "Why Cloud? - A Review of Cloud Adoption Determinants in Organizations." ECIS, 2015.

[2] NIST "NIST Definition of cloud computing v15," National Institute of Standards and Technology: Gaithersburg, MD, 2009.

[3] Kim, W., "Cloud Computing: Today and Tomorrow," Journal of Object Technology, 8(1), 2009, pp. 65-72.

[4] Oliveira, T., Thomas, M., and Espadanal, M., "Assessing the determinants of cloud computing adoption: An analysis of the manufacturing and services sectors, " Information \& Management, 51(5), 2014, pp. 497-510.

[5] Alshamaila, Y., Papagiannidis, S., and Li, F., "Cloud computing adoption by SMEs in the north east of England: A multi-perspective framework," Journal of Enterprise Information Management, 26(3), 2013, pp. 250-275.

[6] Low, C., Chen, Y., and Wu, M., "Understanding the determinants of cloud computing adoption," Industrial Management \& Data Systems, 111(7), 2011, pp. 10061023.

[7] Phaphoom, N., Wang, X., Samuel, S., Helmer, S., \& Abrahamsson, P., "A survey study on major technical barriers affecting the decision to adopt cloud services," Journal of Systems and Software, 103, 2015, pp. 167-181.
[8] Wu, Y., Cegielski, C. G., Hazen, B. T., and Hall, D. J., "Cloud computing in support of supply chain information system infrastructure: understanding when to go to the cloud," Journal of Supply Chain Management, 49(3), 2013, pp. 25-41.

[9] Hsu, P. F., Ray, S., and Li-Hsieh, Y. Y., "Examining cloud computing adoption intention, pricing mechanism, and deployment model," International Journal of Information Management, 34(4), 2014, pp. 474-488.

[10] Lian, J.W., Yen, D.C., Wang, Y.T., "An exploratory study to understand the critical factors affecting the decision to adopt cloud computing in Taiwan hospital, " International Journal of Information Management, 34 (1), 2014, pp. 28-36.

[11] IDC. "Worldwide Broader HPC 2014-2018 Forecast: Servers, Storage, Software, Middleware, and Services. IDC. " 2015.

[12] Geist, A., and Reed, D. A., "A survey of highperformance computing scaling challenges," International Journal of High Performance Computing Applications, 2015, pp. 1-10.

[13] Zenios, S.A., "High-performance computing in finance: The last 10 years and the next," Parallel Computing, 25, 1999, pp. 2149-2175.

[14] Lynn, T., Gourinovitch, A., Kenny, D., and Liang, X., "Drivers and barriers to using high performance computing in the cloud," 2016, http://cloudlightning.eu/market-briefings/ accessed 10 January 2017.

[15] Beck, R. and Toenker, M., "Increasing Dynamic Capabilities through Virtualized Grid-in-Cloud Solutions," International Conference on Information Systems, Orlando, 2012.

[16] Thackston, R. and Fortenberry, R. "High Performance Computing: Considerations When Deciding To Rent Or Buy", SAIS 2015 Proceedings. Pp.16.

[17] Rogers, E. M., "Diffusion of innovations," New York, 1995.

[18] Rogers, E. M., "Elements of diffusion," Diffusion of innovations, 5, 2003, pp. 1-38.

[19] Lin, A., and Chen, N. C., "Cloud computing as an innovation: Perception, attitude, and adoption," International Journal of Information Management, 32(6), 2012, pp. 533-540.

[20] Tornatzky, L., and Fleischer, M., "The process of technology innovation," Lexington, MA. Lexington Books, 1990. 
[21] Gangwar, H., Date, H., and Raoot, A. D., "Review on IT adoption: insights from recent technologies," Journal of Enterprise Information Management, 27(4), 2014, pp. 488-502.

[22] Yusof, M. M., Papazafeiropoulou, A., Paul, R. J., and Stergioulas, L. K., "Investigating evaluation frameworks for health information systems," International Journal of Medical Informatics, 77(6), 2008, pp. 377-385.

[23] Galbraith, J. R., "Organization design: An information processing view, " Interfaces, 4(3), 1974, pp. 28-36.

[24] Melville, N., and Ramirez, R., "Information technology innovation diffusion: An information requirements paradigm," Information Systems Journal, 18(3), 2008, pp. 247-273.

[25] Cegielski, C. G., Allison Jones-Farmer, L., Wu, Y., and Hazen, B. T., "Adoption of cloud computing technologies in supply chains: An organizational information processing theory approach," The International Journal of Logistics Management, 23(2), 2012, pp. 184-211.

[26] Davis, F. D., "Perceived usefulness, perceived ease of use, and user acceptance of information technology," MIS Quarterly, 1989, pp. 319-340.

[27] Chen, L., and Tan, J., "Technology adaptation in ecommerce: Key determinants of virtual stores acceptance," European Management Journal, 22(1), 2004, pp. 74-86.

[28] Gupta, P., Seetharaman, A., and Raj, J. R., "The usage and adoption of cloud computing by small and medium businesses," International Journal of Information Management, 33(5), 2013, pp. 861-874.

[29] Hazen, B. T., Wu, Y., Sankar, C. S., and Jones-Farmer, L. A., "A proposed framework for educational innovation dissemination," Journal of Educational Technology Systems, 40(3), 2012, pp. 301-321.

[30] Agarwal, R., and Prasad, J., "A conceptual and operational definition of personal innovativeness in the domain of information technology," Information Systems Research, 9(2), 1998, pp. 204-215.

[31] Premkumar, G., and Roberts, M., "Adoption of new information technologies in rural small businesses," Omega, 27(4), 1999, pp. 467-484.

[32] Zhu, K., Kraemer, K. L., and Xu, S. "The process of innovation assimilation by firms in different countries: a technology diffusion perspective on e-business," Management Science, 52(10), 2006, pp. 1557-1576.
[33] Chen, D., and Zhao, H., "Data security and privacy protection issues in cloud computing," In International Conference on Computer Science and Electronics Engineering (ICCSEE), IEEE, 1, 2012, pp. 647-651.

[34] Lee, S., and Kim, K. J., "Factors affecting the implementation success of Internet-based information systems," Computers in Human Behavior, 23(4), 2007, pp. 1853-1880.

[35] Hayes, B., "Cloud computing," Communications of the ACM, 51(7), 2008, pp.9-11.

[36] Chang, I. C., Hwang, H. G., Hung, M. C., Lin, M. H., and Yen, D. C., "Factors affecting the adoption of electronic signature: Executives' perspective of hospital information department," Decision Support Systems, 44(1), 2007, pp. 350-359.

[37] Iacovou, C. L., Benbasat, I., and Dexter, A. S., "Electronic data interchange and small organizations: Adoption and impact of technology," MIS Quarterly, 1995, pp. 465-485.

[38] Kuan, K. K., and Chau, P. Y., "A perception-based model for EDI adoption in small businesses using a technology-organization-environment

framework," Information \& Management, 38(8), 2001, pp.507-521.

[39] Marston, S., Li, Z., Bandyopadhyay, S., Zhang, J., and Ghalsasi, A., "Cloud computing-The business perspective," Decision Support Systems, 51(1), 2011, pp. 176-189.

[40] Feldman, M., "China tops supercomputer rankings with new 93-petaflop machine," https://www.top500.org/news/china-topssupercomputer-rankings-with-new-93-petaflopmachine/ accessed 3 March 2017

[41] Soliman, K. S., and Janz, B. D., "An exploratory study to identify the critical factors affecting the decision to establish Internet-based interorganizational information systems," Information \& Management, 41(6), 2004, pp. 697-706.

[42] Baruch, Y., "Response rate in academic studies-A comparative analysis. Human Relations," 52(4), 1999, pp. 421-438.

[43] Wilcox, J. B., Bellenger, D. N., and Rigdon, E. E., "Assessing sample representativeness in industrial survey," Journal of Business \& Industrial Marketing, 9(2), 1994, pp.51-61.

[44] Hair, J. F., Black, W. C., Babin, B. J., and Anderson, R. E., "Multivariate data analysis: A global perspective." Pearson: Upper Saddle River, NJ, 2010. 